PESCE INFESTATO DA ANISAKIS SPP.: COMPORTAMENTO ISPETTIVO ALLA LUCE DEL REG. (CE) N. 1069/2009

\title{
FISH INFESTED WITH ANISAKIS SPP.: INSPECTION BEHAVIOUR IN TERMS OF REG. (CE) N. 1069/2009
}

Sola D. ${ }^{1}$, Federici C. ${ }^{2}$

${ }^{1}$ Istituto Zooprofilattico Sperimentale dell'Umbria e delle Marche, Perugia

${ }^{2}$ Medico Veterinario Libero Professionista, Terni

SUMMARY

KEYWORDS

\begin{abstract}
The inspection behaviour on the fish infested with Anisakis spp., under Regulation (CE) n.1774/2002, was always discordant among those who considered him as a secondary product of category 1 and those who categorize like 2. In near future, Regulation (CE) n.1069/2009 will define that the parasitic diseases are to be included in zoonoses to make attention and definitively poses fish infested with Anisakis in category 1 material.
\end{abstract}

anisakis , category 1 material , European Regulation n. 1069/2009
Il Regolamento (CE) n. 1774/2002 recante norme sanitarie relative ai sottoprodotti di origine animale non destinati al consumo umano, lascia ampio margine di interpretazione ai fini della categorizzazione dei prodotti ittici parassitati da larve di Anisakis spp. Secondo alcuni, infatti, il caso in questione rientra nella definizione di materiale di categoria 1 in quanto viene considerato come sottoprodotto di un animale selvatico, secondo la definizione dell'Art. 2 "animale non detenuto dall'uomo", sospetto di essere affetto da una malattia trasmissibile all'uomo o agli animali (Art. 4, comma 1, lettera a, punto v). Altri, non trovando specifici riferimenti alla parassitosi in oggetto, categorizzano il sottoprodotto come materiale di categoria 2 perché non appartenente né alla categoria 1 né alla categoria 3 (Art. 5,comma1, lettera g). A decorrere dal 4 marzo 2011 entra in vigore il Regolamento (CE) n.1069/2009 recante norme sanitarie relative ai sottoprodotti di origine animale e ai prodotti derivati non destinati al consumo umano, che pone netta chiarezza in merito. Tale regolamento considera opportuno adottare, a bordo dei pescherecci, misure in merito ai rischi nella manipolazione e nello smaltimento del materiale derivato dalla eviscerazione del pesce che manifesta sintomi di malattie, specificando che tali misure devono essere supportate da una idonea valutazione dei rischi al fine di combattere la diffusione di zoonosi, in particolare alcune parassitosi. Quanto appena esposto viene ulteriormente chiarito nell'ambito di applicazione di cui all'Art.2, punto 2, lettera h) “...malattie trasmissibili all'uomo, incluse le parassitosi". Il pesce parassitato da Anisakis spp. dovrà essere considerato definitivamente come materiale di categoria 1 in quanto all'Articolo 8, lettera a), trova spazio nella definizione di "corpi interi $e$ tutte le loro parti, incluse le pelli degli animali seguenti.."(punto v) "animali selvatici, se si sospetta che siano affetti da malattie trasmissibili all'uomo o agli animali". In conclusione, detto comportamento ispettivo che fa seguito a quanto espresso nel Reg. (CE) n. 853/04 (All. III, Sez. VIII, Cap. V, lettera D) che recita : " Gli operatori non devono immettere sul mercato per il consumo umano $i$ prodotti della pesca manifestamente infestati da parassiti”, sarà uniformato. Auspichiamo che anche la dicitura "manifestamente infestati" venga il prima possibile quantificata e normata. 


\section{BIBLIOGRAFIA}

1. Regolamento (CE) n. 1774/2002 recante norme sanitarie relative ai sottoprodotti di origine animale non destinati al consumo umano.

2. Regolamento (CE) n. 1069/2009 recante norme sanitarie relative ai sottoprodotti di origine animale e ai prodotti derivati non destinati al consumo umano e che abroga il regolamento (CE) n. 1774/2002 (regolamento sui sottoprodotti di origine animale).

3. Regolamento (CE) n. 853/2004 che stabilisce norme specifiche in materia di igiene per gli alimenti di origine animale. 